Allan Linneberg, Ching-Ti Liu, Dajiang J. Liu, Yongmei Liu, Ken S. Lo, Artitaya Lophatananon, Andrew J. Lotery, Anu Loukola, Jian'an Luan, Steven A. Lubitz, Leo-Pekka Lyytikäinen, Satu Männistö, Gaëlle Marenne, Angela L. Mazul, Mark I. McCarthy, Roberta McKean-Cowdin, Sarah E. Medland, Karina Meidtner, Lili Milani, Vanisha Mistry, Paul Mitchell, Karen L. Mohlke, Leena Moilanen, Marie Moitry, Grant W. Montgomery, Dennis O. Mook-Kanamori, Carmel Moore, Trevor A. Mori, Andrew D. Morris, Andrew P. Morris, Martina Müller-Nurasyid, Patricia B. Munroe, Mike A. Nalls, Narisu Narisu, Christopher P. Nelson, Matt Neville, Sune F. Nielsen, Kjell Nikus, Pål R. Njølstad, Børge G. Nordestgaard, Dale R. Nyholt, Jeffrey R. O'Connel, Michelle L. O'Donoghue, Loes M. Olde Loohuis, Roel A. Ophoff, Katharine R. Owen, Chris J. Packard, Sandosh Padmanabhan, Colin N. A. Palmer, Nicholette D. Palmer, Gerard Pasterkamp, Aniruddh P. Patel, Alison Pattie, Oluf Pedersen, Peggy L. Peissig, Gina M. Peloso, Craig E. Pennell, Markus Perola, James A. Perry, John R. B. Perry, Tune H. Pers, Thomas N. Person, Annette Peters, Eva R. B. Petersen, Patricia A. Peyser, Ailith Pirie, Ozren Polasek, Tinca J. Polderman, Hannu Puolijoki, Olli T. Raitakari, Asif Rasheed, Rainer Rauramaa, Dermot F. Reilly, Frida Renström, Myriam Rheinberger, Paul M. Ridker, John D. Rioux, Manuel A. Rivas, David J. Roberts, Neil R. Robertson, Antonietta Robino, Olov Rolandsson, Igor Rudan, Katherine S. Ruth, Danish Saleheen, Veikko Salomaa, Nilesh J. Samani, Yadav Sapkota, Naveed Sattar, Robert E. Schoen, Pamela J. Schreiner,

Matthias B. Schulze, Robert A. Scott, Marcelo P. Segura-Lepe, Svati H. Shah, Wayne H.-H. Sheu, Xueling Sim, Andrew J. Slater, Kerrin S. Small, Albert V. Smith, Lorraine Southam, Timothy D. Spector, Elizabeth K. Speliotes, John M. Starr, Kari Stefansson, Valgerdur Steinthorsdottir, Kathleen E. Stirrups, Konstantin Strauch,

Heather M. Stringham, Michael Stumvoll, Liang Sun, Praveen Surendran, Amy J. Swift, Hayato Tada, Katherine E. Tansey, Jean-Claude Tardif, Kent D. Taylor, Alexander Teumer, Deborah J. Thompson, Gudmar Thorleifsson, Unnur Thorsteinsdottir, Betina H. Thuesen, Anke Tönjes, Gerard Tromp, Stella Trompet, Emmanouil Tsafantakis, Jaakko Tuomilehto,

Anne Tybjaerg-Hansen, Jonathan P. Tyrer, Rudolf Uher, André G. Uitterlinden, Matti Uusitupa, Sander W. Laan, Cornelia M. Duijn, Nienke Leeuwen, Jessica van Setten, Mauno Vanhala, Anette Varbo, Tibor V. Varga, Rohit Varma, Digna R. Velez Edwards, Sita H. Vermeulen, Giovanni Veronesi, Henrik Vestergaard, Veronique Vitart, Thomas F. Vogt, Uwe Völker, Dragana Vuckovic, Lynne E. Wagenknecht, Mark Walker, Lars Wallentin, Feijie Wang, Carol A. Wang, Shuai Wang, Yiqin Wang, Erin B. Ware, Nicholas J. Wareham, Helen R. Warren, Dawn M. Waterworth, Jennifer Wessel, Harvey D. White, Cristen J. Willer, James G. Wilson, Daniel R. Witte, Andrew R. Wood, Ying Wu, Hanieh Yaghootkar, Jie Yao, Pang Yao, Laura M. Yerges-Armstrong, Robin Young, Eleftheria Zeggini $\mathbb{D}_{0}$, Xiaowei Zhan, Weihua Zhang, Jing Hua Zhao, Wei Zhao (D), Wei Zhao, Wei Zhou, Krina T Zondervan (D), CHD Exome+ Consortium, EPIC-CVD Consortium, ExomeBP Consortium, Global Lipids Genetic Consortium, GoT2D Genes Consortium, EPIC InterAct Consortium, INTERVAL Study, ReproGen Consortium, T2D-Genes Consortium, The MAGIC Investigators, Understanding Society Scientific Group, Jerome I. Rotter, John A. Pospisilik (1D), Fernando Rivadeneira (D), Ingrid B. Borecki, Panos Deloukas (1), Timothy M. Frayling, Guillaume Lettre, Kari E. North, Cecilia M. Lindgren, Joel N. Hirschhorn and Ruth J. F. Loos

Correction to: Nature Genetics https://doi.org/10.1038/s41588-017-0011-x; published online 26 February 2018.

In the version of this article originally published, one of the two authors with the name Wei Zhao was omitted from the author list and the affiliations for both authors were assigned to the single Wei Zhao in the author list. In addition, the ORCID for Wei Zhao (Department of Biostatistics and Epidemiology, Perelman School of Medicine, University of Pennsylvania, Philadelphia, PA, USA) was incorrectly assigned to author Wei Zhou. The errors have been corrected in the HTML and PDF versions of the article.

\title{
Publisher Correction: BRD4 interacts with NIPBL and BRD4 is mutated in a Cornelia de Lange-like syndrome
}

Gabrielle Olley, Morad Ansari, Hemant Bengani, Graeme R. Grimes, James Rhodes, Alex von Kriegsheim, Ana Blatnik, Fiona J. Stewart, Emma Wakeling, Nicola Carroll, Alison Ross, Soo-Mi Park, Deciphering Developmental Disorders Study, Wendy A. Bickmore (1), Madapura M. Pradeepa (i) and David R. FitzPatrick (1)

Correction to: Nature Genetics; https://doi.org/10.1038/s41588-018-0042-y; Article published online 29 January 2018.

In the version of this article initially published, Wendy Bickmore and Madapura Pradeepa were incorrectly not indicated as corresponding authors. The error has been corrected in the HTML and PDF versions of the paper.

Published online: 12 February 2018

https://doi.org/10.1038/s41588-018-0069-0 